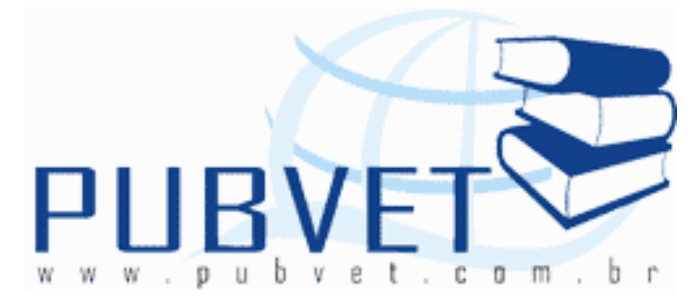

PUBVET, Publicações em Medicina Veterinária e Zootecnia.

\title{
Índices de conforto térmico aplicado a animais de produção
}

Geovan Figueirêdo de Sá Filho ${ }^{1}$, Janio Lopes Torquato ${ }^{1}$, João Batista Freire de Souza Junior ${ }^{1}$, Hérica Girlane Tertulino Domingos ${ }^{1}$, Leonardo Lelis de Macedo Costa $^{1}$, Maiko Roberto Tavares Dantas ${ }^{1}$

${ }^{1}$ Universidade Federal Rural do Semi-Árido, Departamento de Ciências Animais, Laboratório de Biometeorologia, Biofísica Ambiental e Bem-Estar Animal (LABBEA), BR 110, Km 47, Costa e Silva, CEP: 59625-900, Mossoró-RN.

\section{Resumo}

Trocas de energia térmica entre os animais e o meio ambiente dependem de fatores fisiológicos e ambientais, que estão relacionados a aspectos complexos e variados. Um animal sofre estresse térmico quando produz mais calor do que pode dissipar. Para se ajustar, ele é obrigado a reduzir o consumo de alimentos e sua produção necessariamente declina. Em regiões tropicais a radiação solar é de alta magnitude e a temperatura média radiante do ambiente durante o dia é normalmente muito mais elevada do que a do ar, e a capacidade dos animais para lidar depende de algumas características físicas. Existem diversos índices cujo objetivo fundamental é determinar a adequação de um ambiente com relação a uma atividade ou a um tipo específico de animal. Tais índices dificilmente podem ser de uso generalizado, uma vez que são estruturados com base em determinados fatores ambientais, que podem ser importantes para alguns animais e não para outros. Além disso, certos 
SÁ FILHO, G.F. et al. Índices de conforto térmico aplicado a animais de produção. PUBVET, Londrina, V. 6, N. 14, Ed. 201, Art. 1349, 2012.

índices são baseados em associações específicas de fatores ambientais, que ocorrem em locais determinados.

Palavras-chave: ambiente térmico, estresse térmico, produção animal

\title{
Thermal comfort indices applied to livestock
}

\begin{abstract}
Exchanges of heat between the animals and the environment depend on physiological factors and environmental aspects that are related to complex and varied. An animal suffer heat stress when it produces more heat than it can dissipate. To fit, he is forced to reduce consumption of foods and their production necessarily declines. In tropical solar radiation is of high magnitude and the mean temperature of the heating environment during the day is usually much higher than that of air, and the ability to handle animals depends on certain physical characteristics. There are several indexes whose primary goal is to determine the suitability of an environment with respect to an activity or a specific type of animal. These indices can hardly be widely used, since they are structured on the basis of certain environmental factors, that may be important in some animals and not for others. Furthermore, some indices are based on specific associations of environmental factors that occur in certain places.
\end{abstract}

Keywords: thermal environment, heat stress, animal production

\section{INTRODUÇÃO}

As condições ambientais existentes nos países situados nos trópicos, como é o caso do Brasil, causam problemas na criação de animais, em geral, devido ao fato de apresentarem médias altas de temperatura durante o ano, causando o chamado stress térmico. Um animal sofre stress térmico quando produz mais calor do que pode dissipar. Para se ajustar, ele é obrigado a reduzir o consumo de alimentos e sua produção necessariamente declina (BOND, 1954). 
SÁ FILHO, G.F. et al. Índices de conforto térmico aplicado a animais de produção. PUBVET, Londrina, V. 6, N. 14, Ed. 201, Art. 1349, 2012.

Desde que foram reconhecidas as diferenças entre os animais quanto a sua capacidade de enfrentar as variações climáticas, têm sido feitas tentativas para estabelecer critérios de seleção dos mais aptos para ambientes específicos, além de se classificar os diversos tipos de ambientes sob o ponto de vista do conforto desses animais. Em sua maioria, esses índices destinamse à classificação de ambientes, mas há alguns que avaliam diretamente os animais. (SILVA, 2000)

Existem diversos índices cujo objetivo fundamental é determinar a adequação de um ambiente com relação a uma atividade ou a um tipo específico de animal. Tais índices dificilmente podem ser de uso generalizado, uma vez que são estruturados com base em determinados fatores ambientais, que podem ser importantes para alguns animais e não para outros. Além disso, certos índices são baseados em associações específicas de fatores ambientais, que ocorrem em locais determinados. (SILVA, 2000)

\section{2 ÍNDICE DE ESTRESSE DE CALOR DE BELDING-HATCH}

Desenvolvido por Belding \& Hatch (1995) para o uso em seres humanos, o índice consiste na relação entre a evaporação requerida $E_{\text {req }}$ e a evaporação máxima $E_{\max }$ :

$\mathrm{HSI}=\mathrm{E}_{\mathrm{req}} / \mathrm{E}_{\max }$

Já que a evaporação respiratória é desconsiderada nesse índice, o próprio não pode ser utilizado para animais nos quais esse mecanismo seja de fundamental importância para a dissipação do calor.

\section{3 ÍNDICE DE GLOBO ÚMIDO}

Refere-se a um índice do estresse térmico total de uma dada combinação de fatores ambientais sobre um indivíduo, tendo sido proposto originalmente por Yaglou \& Minard (1957). É expresso por:

WBGT $=0,7 \mathrm{t}_{\mathrm{w}}+0,2 \mathrm{t}_{\mathrm{g}}+0,1 \mathrm{t}_{\mathrm{a}}$

Sendo, $t_{w}$ a temperatura do bulbo úmido medida em um psicrômetro exposto à radiação solar direta, mas não ventilado artificialmente; $t_{a}$ e $t_{g}$ são as 
SÁ FILHO, G.F. et al. Índices de conforto térmico aplicado a animais de produção. PUBVET, Londrina, V. 6, N. 14, Ed. 201, Art. 1349, 2012.

temperaturas do ar e do globo de Vernon, respectivamente, ambas em graus ${ }^{\circ} \mathrm{C}$.

\section{4 ÍNDICE DE TEMPERATURA E UMIDADE}

Thom em 1958 desenvolveu originalmente um índice de conforto térmico humano; Em sua forma mais conhecida, apresenta-se como:

$\mathrm{THI}=\mathrm{t}_{\mathrm{a}}+0,36 \mathrm{t}_{\mathrm{po}}+41,5$

Onde, $t_{a}$ é a temperatura do ar e $t_{p o}$ é a temperatura de ponto de orvalho, ambos em graus celsius $\left({ }^{\circ} \mathrm{C}\right)$.

Esse índice tem sido usado para descrever o conforto térmico de animais. De acordo com Hahn (1985), um valor de THI igual a 70 ou menos indica condição normal, não estressante; um valor entre 71 e 78 é crítico; entre 79 e 83, indica perigo; acima de 83 já constitui uma emergência.

\section{5 ÍNDICES DE GLOBO E UMIDADE}

Um dos mais importantes fatores ambientais é a radiação térmica, principalmente para animais situados em campo aberto. Mas se o índice de temperatura e umidade (THI) for utilizado para avaliar o ambiente, não demonstrará quaisquer diferenças para animais mantidos em interiores, à sombra ou sob o sol direto. Ponderando isso, Buffingtion et al. (1981) propuseram para vacas leiteiras uma modificação do THI, que denominaram índice de globo e umidade (black Globe-Humidity Index, BGHI ou ITGU), $\mathrm{BGHI}=\mathrm{t}_{\mathrm{g}}+0,36 \mathrm{t}_{\mathrm{po}}+41,5$

Em que, $\mathrm{t}_{\mathrm{g}}$ é a temperatura do globo negro $\left({ }^{\circ} \mathrm{C}\right)$.

Buffington et al. (1981) asseguraram que o ITGU seria um indicador mais preciso do conforto térmico e da produção animal quando comparado ao ITU em condições ambientais onde a radiação solar ou a movimentação do ar sejam altas, sendo que sob condições moderadas de radiação solar são igualmente eficientes, e quando se compara medições em locais com e sem cobertura, os ITUs não apresentaram diferenças significativas, enquanto que o 
SÁ FILHO, G.F. et al. Índices de conforto térmico aplicado a animais de produção. PUBVET, Londrina, V. 6, N. 14, Ed. 201, Art. 1349, 2012.

ITGU apresentou diferenças significativas, principalmente para locais expostos a radiação direta.

\section{6 ÍNDICE DE ESTRESSE TÉRMICO DE GIVONI}

Considerando a troca de calor sensível entre a epiderme e o ambiente, a temperatura da epiderme varia conforme a equação:

$t_{s}=35+6\left(t_{0}-35\right)$

Sendo, $t_{0}$ a temperatura operativa $\left({ }^{\circ} \mathrm{C}\right)$

O índice não foi desenvolvido para animais, mas sua utilização em equinos é viável, já que para essa espécie a sudação é quase tão importante quanto para seres humanos (GIVONI, 1963).

\section{7 ÍNDICE DE CONFORTO TÉRMICO PARA OVINOS}

Índice desenvolvido visando especificamente a classificação de ambientes para a criação de ovinos (SILVA \& BARBOSA, 1993), sendo:

$\mathrm{TCI}=0,659 \mathrm{t}_{\mathrm{a}}+0,511 \mathrm{p}+0,550 \mathrm{t}_{\mathrm{g}}-0,042 \mathrm{v}$

Onde, $t_{a}$ é a temperatura do $\operatorname{ar}\left({ }^{\circ} \mathrm{C}\right)$, $\mathrm{p}$ a pressão parcial de vapor $(\mathrm{kPa}), \mathrm{t}_{\mathrm{g}} \mathrm{a}$ temperatura de um globo de Vernon $\left({ }^{\circ} \mathrm{C}\right)$ e $v$ a velocidade do vento $\left(\mathrm{m} . \mathrm{s}^{-1}\right)$.

\section{8 ÍNDICE DE TEMPERATURA EQUIVALENTE PARA VACAS}

Baeta et al. (1987) combinaram os efeitos da temperatura, umidade e velocidade do vento sobre o equilíbrio térmico de vacas leiteiras em uma equação que denominaram de índice de temperatura equivalente, expresso em graus ${ }^{\circ} \mathrm{C}$ :

$\mathrm{ETI}=27,88-0,456 \mathrm{t}_{\mathrm{a}}+0,010754 \mathrm{t}-0,4905 \mathrm{U}+0,00088 \mathrm{U}^{2}+1,1507 \mathrm{~V}-$ $0,126447 \mathrm{~V}^{2}+0,019876 \mathrm{Ut}_{\mathrm{a}}-0,046313 \mathrm{Vt}_{\mathrm{a}}$

Sendo, $t_{a}$ é a temperatura do $\operatorname{ar}\left({ }^{\circ} \mathrm{C}\right), \mathrm{U}$ a umidade relativa $(\%)$ e $\mathrm{V}$ a velocidade do vento $\left(\mathrm{m} \cdot \mathrm{s}^{-1}\right)$.

Assim, puderam obter, nas condições estudadas, as escalas de risco a seguir que podem ser consideradas para os animais, conforme o valor de ETI: 
SÁ FILHO, G.F. et al. Índices de conforto térmico aplicado a animais de produção. PUBVET, Londrina, V. 6, N. 14, Ed. 201, Art. 1349, 2012.

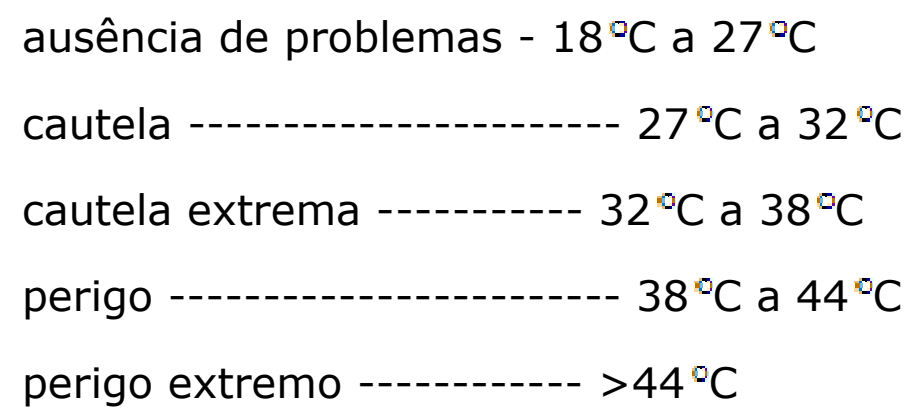

\section{9 ÍNDICE DE TOLERÂNCIA AO CALOR}

\section{1 ÍNDICE DE BACCARI JR}

Índice baseado em medições feitas nos animais. Diferentemente dos outros índices que avaliam apenas variáveis climáticas como temperatura do ar e umidade relativa, o ITC avalia o grau de tolerância de um animal considerando somente um parâmetro fisiológico ou por combinações feitas com parâmetros ambientais.

Existem múltiplos índices que avaliam o nível de tolerância ao calor dos animais, variando segundo autor e espécie animal. Os mais notórios e utilizados, estão o Índice de Baccari Jr. (1986) que consiste uma mensuração da temperatura retal, onde os animais em repouso são colocados na sombra, durante um período de duas horas, em seguida são expostos à radiação solar direta, durante uma hora e em seguida retornam à sombra, onde permanecem por igual período de tempo quando a segunda mensuração da temperatura retal deve ser feita. Expressa da seguinte forma:

ITC $=10-\left(t_{r 2}-t_{r 1}\right)$, onde:

$t_{r 2}:$ temperatura retal ao sol;

$\mathrm{t}_{\mathrm{r} 1}$ : temperatura retal à sombra. 
SÁ FILHO, G.F. et al. Índices de conforto térmico aplicado a animais de produção. PUBVET, Londrina, V. 6, N. 14, Ed. 201, Art. 1349, 2012.

As diferenças entre as temperaturas retais resultam em um índice de 0 à 10, que quanto mais próxima de 10 indica maior capacidade de perda de calor adquirido através da radiação solar.

\section{2 ÍNDICE DE IBÉRIA}

Proposto por Rhoad (1940) utiliza somente valores de temperatura retal, medidas duas vezes. Dessa forma, temos:

ITC $=100-18\left(t_{m}-38,33\right)$, onde:

$t_{m}:$ temperatura retal média

Os valores variam de 0 a 100, e mostram que quanto mais próximo de 100 indica maior resistência às condições tropicais.

\section{3 ÍNDICE DE RAUSCHENBACH-YEROKHIN}

Rauschenbach-Yerokhin (1975) combinaram os valores de temperatura do ar e temperaturas retais.

ITC $=\left(1,2 \times t_{a}\right)-(20 \times d)+52$, onde:

$t_{a}$ : temperatura do $\operatorname{ar}\left({ }^{\circ} \mathrm{C}\right)$;

d: diferença entre as temperaturas retais.

A utilização de testes bioclimáticos aplicáveis em nível de campo é uma alternativa importante e necessária na escolha de animais adaptados às condições tropicais.

\section{0 ÍNDICE DE ESTRESSE TÉRMICO PARA VACAS LEITEIRAS EM REGIÕES INTERTROPICAIS}

Foram utilizadas 178 vacas leiteiras para o experimento, sendo medidas a temperatura retal $\left(\mathrm{TR},{ }^{\circ} \mathrm{C}\right)$, a taxa respiratória (FR, resp. / Min.), a 
SÁ FILHO, G.F. et al. Índices de conforto térmico aplicado a animais de produção. PUBVET, Londrina, V. 6, N. 14, Ed. 201, Art. 1349, 2012.

temperatura superfície da camada de pêlos $\left(\mathrm{TS},{ }^{\circ} \mathrm{C}\right.$ ), e a taxa de sudação ( $\mathrm{SW}$, $\mathrm{GM}^{-2} \cdot \mathrm{h}^{-1}$ ) em duas regiões do Brasil, uma com condições subtropicais de Jaboticabal - SP e a outra na região semi-árida equatorial de Limoeiro do Norte, CE. A Tabela 1 mostra os valores médios das variáveis ambientais durante as observações.

Tabela 1 Os valores médios das variáveis ambientais e fisiológicas durante as observações na região subtropical (SBT) e em cinco rebanhos da região semiárida (Sar1 para Sar5).

\begin{tabular}{lllllll}
\hline Rebanho & Sar1 & Sar2 & Sar3 & Sar4 & Sar5 & Sbt \\
\hline $\boldsymbol{T}_{\boldsymbol{A}}\left({ }^{\circ} \mathbf{C}\right)$ & 32.4 & 34.8 & 33.6 & 33.0 & 31.6 & 27.2 \\
$\boldsymbol{T}_{\boldsymbol{G}}\left({ }^{\circ} \mathbf{C}\right)$ & 44.7 & 47.9 & 42.3 & 41.6 & 49.0 & 28.5 \\
$\boldsymbol{U}\left(\mathbf{m} \cdot \mathbf{s}^{-1}\right)$ & 0.71 & 0.83 & 3.08 & 3.99 & 1.58 & 0.60 \\
$\boldsymbol{P}_{\boldsymbol{V}}(\mathbf{k P a})$ & 3.11 & 3.14 & 2.86 & 2.64 & 2.75 & 1.70 \\
$\boldsymbol{T}_{\boldsymbol{R}}\left({ }^{\circ} \mathbf{C}\right)$ & 39.6 & 40.6 & 38.5 & 38.7 & 38.8 & 39.2 \\
$\boldsymbol{F}_{\boldsymbol{R}}(\mathbf{b r e a t h s} / \mathbf{m i n})$ & 84 & 107 & 49 & 38 & 57 & 36 \\
$\boldsymbol{T}_{\boldsymbol{S}}\left({ }^{\circ} \mathbf{C}\right)$ & 41.2 & 44.8 & 37.8 & 40.8 & 45.4 & 32.9 \\
$\boldsymbol{S}_{\boldsymbol{W}}\left(\mathbf{g} \cdot \mathbf{m}^{-\mathbf{2}} \cdot \mathbf{h}^{-\mathbf{1}}\right)$ & 64 & 81 & 210 & 252 & 121 & 127 \\
\hline
\end{tabular}

As observações em Jaboticabal, foram feitas no período de agosto a dezembro, 1-2 dias por semana, 11:00-15:00 horas, depois que as vacas permaneceram expostas ao sol, em Limoeiro as vacas permaneceram exposta ao sol no período de 07:00-11:00 horas, sendo observado de abril a julho, no período de meio-dia até 16:00 horas. As variáveis ambientais foram mensuradas nos mesmos lugares que estavam os animais. Não foram consideradas vacas leiteiras, sendo escolhidos de forma aleatória em cada rebanho. Essas vacas eram predominantemente negras ( $>74 \%$ da superfície do corpo) como a maioria das raça Holandesa no Brasil, com pêlos muito curtos (pelagem $<3 \mathrm{~mm}$ ).

A estimativa do índice de stress térmico foi feito como se segue. Primeiro, foi calculada a correlação canônica entre dois conjuntos de variáveis ( $X$ para o meio ambiente e $Y$ para as medições fisiológicas), de acordo com os 
SÁ FILHO, G.F. et al. Índices de conforto térmico aplicado a animais de produção. PUBVET, Londrina, V. 6, N. 14, Ed. 201, Art. 1349, 2012.

procedimentos descritos por Rencher (1995). As quatro características fisiológicas são então resumidos em apenas uma variável por meio de uma análise de componente principal. Considerando a matriz de correlação das características fisiológicas (RYY), o autovetor associado ao seu maior autovalor $\mathrm{y}_{1}$ é, cujos elementos correspondem a TR, FR, TS e SW, respectivamente. $O$ primeiro componente principal será dada como:

$y_{1}=e_{11} \frac{T_{R}-\overline{T_{R}}}{\sigma_{T_{R}}}+e_{21} \frac{F_{R}-\overline{F_{K}}}{\sigma_{\overline{F_{R}}}}+e_{31} \frac{T_{S}-\overline{T_{S}}}{\sigma_{T_{S}}}+e_{41} \frac{S_{W}-\overline{S_{W}}}{\sigma_{S W}}$

Os valores calculados y 1 foram tomadas como variáveis dependentes e diferentes combinações de variáveis ambientais como as independentes, então, uma série de índices de conforto térmico foram determinados por correlações múltiplas. As respectivas funções foram comparadas entre si por seus valores de R2 e pelas respectivas correlações com as características fisiológicas. $O$ índice a seguir foi finalmente escolhido:

$$
\begin{gathered}
\text { ITSC }=7.95053+0,0667 T_{G}+0.0673 U+0.0214 U^{2}-1.9005 P_{V}+0.1749 P_{V}^{2} \\
+\quad+0.045 T_{A} P_{V}-0.0095 T_{G} U
\end{gathered}
$$

com um coeficiente de determinação $R 2=0,734$.

Os valores ITSC obtidos pela aplicação da equação (5) para os dados de campo foram correlacionados com as variáveis medidas nos animais, mostrando elevados coeficientes significativas $(P<0,01)$ de correlação: $\operatorname{tr}=$ $0,472, \mathrm{FR}=0,793, \mathrm{TS}=0,755$ e $\mathrm{SW}=0,570$.

Quatro níveis ITSC podem ser sugeridos em termos de estresse térmico: $\leq 8$ conforto, 8-10 estresse ameno, 10-11 estresse moderado, $>11$ muito angustiante. Ao testar o novo índice de 1.321 dados, os resultados apresentaram valores médios de 9,9 a 10,9 para os locais semi-áridas; e 8,7 para o único local subtropical. Apesar valores de índice tão elevadas como 10,5 , vacas não apresentaram sinais significativos ofegante, e da temperatura rectal variou entre 38,5 e $40^{\circ} \mathrm{C}$. Na Figura 1 mostra-se claramente que a semi-áridas locais com ITSC $>10$ são aquelas caracterizadas pela alta velocidade do vento e baixa TG (Macaíba, São José do Mipibu, Flores, Limoeiro e Quixeramobim; para um local (São João do Jaguaribe) ITSC era inferior a 10, 
SÁ FILHO, G.F. et al. Índices de conforto térmico aplicado a animais de produção. PUBVET, Londrina, V. 6, N. 14, Ed. 201, Art. 1349, 2012.

apesar de que é na mesma região geográfica como Limoeiro, porque o $U$ alta (4 ms-1). Assim, um nível elevado ITSC é uma indicação da necessidade de proteção contra a radiação, usando árvores amplamente distribuídos no campo onde as vacas devem ser manejadas para a produção intensiva, essa habitação deve ser planejada para:. (a) proporcionar uma proteção suficiente contra todas as fontes de radiação térmica e (b) ser amplamente aberto para o ventos, como o uso de dispositivos de aspersão de água e ventiladores elétricos, que estão presentes em algumas explorações leiteiras em Macaíba e São José do Mipibu (RN).

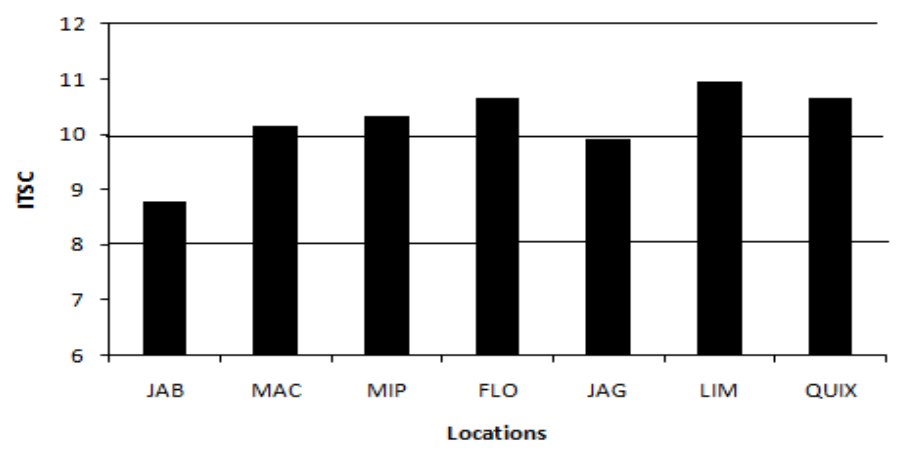

Figura 1 Os valores calculados do índice de conforto térmico (ITSC) para sete locais: Jaboticabal (JAB), Macaíba (MAC), São José do Mipibu (MIP), Flores (FLO), São João do Jaguaribe (JAG), Limoeiro do Norte (LIM ) e Quixeramobim (Quix).

\section{APLICAÇÃo}

\section{1 Índices de temperatura e umidade}

Comprovado por Turco et. al. que definiram o zoneamento bioclimático para o Estado da Bahia através do índice de THI (ou ITU), onde identificaram as regiões estressantes para o gado leiteiro na época mais quente, como também no período de temperaturas consideradas baixas.

Cardoso em 2008, avaliando a termorregulação de ovinos da raça Santa Inês e Dorper, no Meio-norte do Brasil, percebeu que os índices de 
SÁ FILHO, G.F. et al. Índices de conforto térmico aplicado a animais de produção. PUBVET, Londrina, V. 6, N. 14, Ed. 201, Art. 1349, 2012.

temperatura e umidade (ITU) apresentaram-se enquadrados a partir da faixa de alerta, com predominância das faixas de perigo e emergência, segundo as classificações de Hahn (1985).

\section{2 Índice de globo e umidade}

Silva \& Barbosa (1993) aplicaram ambos esses índices em ovinos, confirmando a superioridade do BGHI (ou ITGU) sobre o THI (ou ITU) também para essa espécie.

Já Furtado et. al. (2003), relatou que o BGHI apresentou para frangos de corte, nos horários mais quentes, valores médios considerados acima do ideal, mostrando assim, a precisão desse índice para animais criados em locais que não recebe radiação solar direta.

Enquanto Sampaio et. al. (2004), observou uma redução nos valores e na amplitude do ITGU no interior de uma instalação avícola, principalmente no verão e nas horas mais quentes do dia, assim como a redução da carga térmica radiante em função da instalação foi de até $35 \%$ em relação à área não sombreada.

Navarini et. al. (2009) avaliando o conforto térmico de bovinos da raça nelore a pasto sob diferentes condições de sombreamento e a pleno sol, utilizando o ITGU com índice de conforto, concluiu que a melhor condição térmica foi observada em ambiente de sombreamento proporcionado por pequenos bosques constituídos de árvores de Guajuvira, comparada à proporcionada por árvores isoladas de Goiabeira e Laranjeira, e para exposição a pleno sol.

Já Souza (2011), realizando um estudo sobre o índice de conforto térmico para ovinos e caprinos: índice de temperatura do globo negro e umidade registrada em pesquisas no Brasil, concluiu que há uma correlação positiva do ITGU com a temperatura retal e a frequência respiratória em ovinos e caprinos. Os valores de ITGU considerados estressantes para ovinos e caprinos diferem dos recomendados para bovinos. O valor de ITGU igual a 83 pode 
SÁ FILHO, G.F. et al. Índices de conforto térmico aplicado a animais de produção. PUBVET, Londrina, V. 6, N. 14, Ed. 201, Art. 1349, 2012.

indicar uma condição de estresse médio-alto para ovinos enquanto para caprinos um estresse baixo.

\section{3 Índice de tolerância ao calor}

Souza et. al. (2011) afirmaram que caprinos metiços Boer nos graus de sangue ( $3 / 4$ e $1 / 2$ ) com animais sem raça definida apresentaram o mesmo grau de tolerância ao calor dos caprinos sem raça definida, ambos criados no sistema extensivo no semiárido paraibano.

Titto et al. (1998), estudando a resistência das raças Nelore e Marchigiana ao trópico observaram que a raça italiana apresentou excelente tolerância ao calor juntamente com a raça Nelore, com valores de 9,51 e 9,87 respectivamente, segundo o teste de Baccari Jr. Já Müller, (1982) fazendo uso do teste de Ibéria na avaliação de raças mais tolerantes ao calor, observou melhor desempenho para o Guzerá $(91,2)$ e pior para o Aberdeen Angus.

Souza et. al. (2006) estudando os parâmetros fisiológicos e índice de tolerância ao calor de bovinos da raça sindi no semi-árido paraibano puderam concluir que os bovinos da raça Sindi apresentam alto índice de tolerância ao calor, o que possibilita sua criação em regiões Semi-áridas.

\section{CONCLUSÃO}

O Índice de globo e umidade mostrou-se ser o mais confiável quando usado em regiões que a radiação solar ou movimentação do ar sejam elevadas. Enquanto outros índices encontram-se em desuso, devido não serem tão precisos quanto a informação sobre o conforto térmico dos animais. Já um outro índice que se destacou foi o índice de tolerância ao calor, que é baseado em medições feitas nos animais, diferentemente dos outros índices que avaliam apenas variáveis climáticas como temperatura do ar e umidade relativa, assim, esse índice avalia o grau de tolerância de um animal considerando somente um parâmetro fisiológico ou por combinações feitas com parâmetros ambientais. 
SÁ FILHO, G.F. et al. Índices de conforto térmico aplicado a animais de produção. PUBVET, Londrina, V. 6, N. 14, Ed. 201, Art. 1349, 2012.

O Índice de estresse térmico para vacas leiteiras em regiões intertropicais, sendo um índice recentemente desenvolvido, se mostrou eficiente na observação do nível de estresse de vaca leiteiras no Brasil, para posteriormente serem tomadas medidas que possibilitem um melhor conforto térmico para essas vacas.

De modo geral, cada índice possui sua particularidade, e desse modo, cabe ao autor do estudo em questão utilizar o índice que melhor se adéque ao seu experimento, para que assim possam ser obtidos resultados de relevância para a comunidade científica.

\section{REFERÊNCIAS}

BACCARI JUNIOR, F.; POLASTRE, R.; FRÉ, C. A.; ASSIS, P. S. "Um novo índice de tolerância ao calor para bubalinos: correlação com o ganho de peso". In: REUNIÃO ANUAL DA SOCIEDADE DE ZOOTECNIA, 23., 1986, Campo Grande, MS. Anais... Campo Grande: SBZ, 1986. p. 316.

BAETA, F.C.; MEADOR, N.F.; SHANKLIN, M.D.; JOHNSON, H.D. "Equivalent temperature indexis temperatures above the thermoneutral for lactating dairy cows". Paper pres. Meeting of the ASAE, June 28-July 1, 1987, Baltimore, 21pp, mimeo.

BELDING, H.S.; HATCH, T.F. "Index for evaluating heat stress in terms of resulting physiological strain". Heath Pip. Air Condit. 27:129-136, 1955.

BOND, T.E.; KELLY, C.F.; ITTNER, N.R. "Radiation studies of pointed shade materials". Agricultural Engineering, St. Joseph, v.35, n.6, p.389-392,1954.

BUfFINGTON, D.E.; COLLAZO-AROCHO, A.; CANTON, G.H.; PITT, D.; THATCHER, W.W.; COLLIER, R.J. "Black-Globe-Humidity Index (BGHI) as comfort equation for dairy cows" Transactions of the ASA 24:711-714, 1981.

CARDOSO, F. S. "Termorregulação de ovinos da raça Santa Inês e da raça Dorper no MeioNorte do Brasil" - 2008.

FURTADO, D. A.; AZEVEDO, P. V.; TINÔCO, I. F. F. "Análise do conforto térmico em galpões avícolas com diferentes sistemas de acondicionamento". Revista Brasileira de Engenharia Agrícola e Ambiental, v.7, n.3, p.559-564, 2003. Campina Grande, PB.

GIVONI, B. "Estimation of the effect of climate on man: development of a new thermal index". Res. Report UNESCO. Haifa: Israel Inst. of Technology, 1963.

HAHN, G.L. "Management and housing of farm animals in hot environments". In: Strss physiology in livestoeh (M.K.Yousef, ed), vol.II. Boca raton; CRC Press, 1985.

JÁCOME, I. M. T. D.; FURTADO, D. A.; LEAL, A. F.; SILVA, J. H. V.; MOURA, J. F. P. "Avaliação de índices de conforto térmico de instalações para poedeiras no nordeste do Brasil". Revista Brasileira de Engenharia Agrícola e Ambiental v.11, n.5, p.527-531, 2007. Campina Grande, PB, UAEAg/UFCG.

MÜLLER, P.B. Bioclimatologia Aplicada aos animais domésticos. 2a edição Editora Sulina / Porto Alegre - RS. 1982. 
NAVARINI, F. C.; KLOSOWSKI, E. S.; CAMPOS, A. T.; TEIXEIRA, R. A.; ALMEIDA, C. P. "Conforto térmico de bovinos da raça nelore a pasto sob diferentes condições de sombreamento e a pleno sol". Eng. Agríc., Jaboticabal, v.29, n.4, p.508-517, out./dez. 2009.

RAUSCHENBACH, J.O.; YEROKHIN, P.I. "Quantitative estimation of heat tolerance in animals". Novosibirski Publishing House nauka, 1975.

RENCHER, A.C., 1995: Methods of Multivariate Analysis. Wiley, New York, 627p.

RHOAD, A.O. "The Iberia heat tolerance test for cattle". Tropical Agric. 21:162, 1940.

ROCHA, D. R.; ARAúJO, A. A.; MOURA, A. A. A. N. "Avaliação de estresse térmico em vacas leiteiras mestiças (Bos taurus $x$ Bos indicus) durante os períodos chuvoso e seco no Estado do Ceará." In: REUNIÃO ANUAL DA SOCIEDADE BRASILEIRA DE ZOOTECNIA, 45., 2008, Lavras, Mg. 2008. p. 1 - 3.

SAMPAIO, C. A. P.; CRISTANI, J.; DUBIELA, J. A.; BOFF, C. E.; OliVEIRA, M. A. "Avaliação do ambiente térmico em instalação para crescimento e terminação de suínos utilizando os índices de conforto térmico nas condições tropicais". Ciência Rural, Santa Maria, v.34, n.3, p.785-790, mai-jun, 2004.

SILVA, R. G. "Introdução à Bioclimatologia Animal". São Paulo - Ed.: Nobel 2000.

SILVA, R.G.; BARBOSA, O.R. "Thermal comfort index for sheep". Proc. 13th International Congress of Biometeorology, September 12-18, 1993, Calgary, Part 2, vol.2, pg. 383-392.

SOUZA, B. B.; SILVA, G. A.; FREITAS, M. M. S.; CUNHA, M. G. G.; BeníCiO, T. M. A. "Respostas fisiológicas e índice de tolerância ao calor de caprinos mestiços de boer no semiárido". Revista Verde (Mossoró - RN - Brasil) v.6, n.3, p. 146 - 151 julho/setembro de 2011

SOUZA, B. B.; SILVA, R. M. N.; MARINHO, M. L.; SILVA, G. A.; SILVA, E. M. N.; SOUZA, A. P. "Parâmetros fisiológicos e índice de tolerância ao calor de bovinos da raça sindi no semi-árido paraibano". Ciênc. agrotec., Lavras, v. 31, n. 3, p. 883-888, maio/jun., 2007.

THOM, E.C. "Cooling degree: day air conditioning, heating, and ventilating". Transactions of the Amer. Soc. Heating, Refrigerating and Air-Conditioning Engrs. 55:65-72, 1958.

TITTO, E. A. L. ; VELlOSO, L. ; ZANETTI, M. A. ; CRESTA, A. ; TOledO, L. A. ; MARTINS, J. H. Teste de tolerância ao calor em novilhos Nelore e Marchigiana. Revista Portuguesa de Zootecnia, Vila real/Portugal, v. 5, n.1, p. 67-70, 1998.

TURCO, S. H. N.; SILVA, T. G. F.; SANTOS, L. F. C.; RIBEIRO, P. H. B.; ARAÚJO, G. G. L.; TEIXEIRA, A. H. C.; HOLANDA, E. V.; AGUIAR, M. A. "Zoneamento bioclimático para vacas leiteiras no estado da bahia"

YAGLOU, C.P; MINARD, D. "Control of heat casualties at military training centers". Amer. Med. Assoc. Archs. Ind. Health 16:302-316, 1957. 